\title{
Muséologies
}

Les cahiers d'études supérieures

\section{Le patrimoine de la famille et des entreprises Breakey : la reconnaissance d'un héritage anglo-protestant par la communauté lévisienne}

\section{Sophie Couture Samson}

Volume 3, numéro 1, automne 2008

URI : https://id.erudit.org/iderudit/1033583ar

DOI : https://doi.org/10.7202/1033583ar

Aller au sommaire du numéro

Éditeur(s)

Association Québécoise de Promotion des Recherches Étudiantes en Muséologie (AQPREM)

ISSN

1718-5181 (imprimé)

1929-7815 (numérique)

Découvrir la revue

Citer cet article

Couture Samson, S. (2008). Le patrimoine de la famille et des entreprises Breakey : la reconnaissance d'un héritage anglo-protestant par la communauté lévisienne. Muséologies, 3(1), 110-127. https://doi.org/10.7202/1033583ar

\section{Résumé de l'article}

L'actuel contexte de mondialisation incite les gouvernements à se doter de politiques culturelles et de politiques de protection du patrimoine. Dans cet article, Sophie Couture-Samson présente l'étude qu'elle a réalisée pour la Ville de Lévis, dans le cadre de la mise en oeuvre de sa politique culturelle. Elle y présente des recommandations visant la conservation et la mise en valeur d'éléments patrimoniaux liés à l'histoire de la famille et des entreprises Breakey. 
Article quatre

Le patrimoine de la famille et des entreprises

Breakey: la reconnaissance d'un héritage

anglo-protestant par la communauté lévisienne 
L'actuel contexte de mondialisation incite les gouvernements à se doter de politiques culturelles et de politiques de protection du patrimoine. Dans cet article, Sophie Couture-Samson présente l'étude qu'elle a réalisée pour la Ville de Lévis, dans le cadre de la mise en œuvre de sa politique culturelle. Elle y présente des recommandations visant la conservation et la mise en valeur d'éléments patrimoniaux liés à l'histoire de la famille et des entreprises Breakey.

SOPHIE COUTURE SAMSON EST TITULAIRE D'UN BACCALAURÉAT EN HISTOIRE DE L'UNIVERSITÉ LAVAL. ELLE A ÉGALEMENT OBTENU, EN MARS 2008, UN DIPLÔME DE MAîTRISE EN MUSÉOLOGIE DE L'UNIVERSITÉ DU QUÉBEC À MONTRÉAL. ELLE A ENSUITE RÉALISÉ DES CONTRATS DE RECHERCHE POUR LE MUSÉE DU SKI DES LAURENTIDES ET LE MUSÉE CANADIEN DE LA POSTE. EN SEPTEMBRE 2008, ELLE A ENTREPRIS UN DOCTORAT EN HISTOIRE À LUNIVERSITÉ LAVAL, SOUS LA DIRECTION DES PROFESSEURS PHILIPPE DUBÉ ET JACQUES BERNIER. SON PROJET DE DOCTORAT PORTE SUR LES COLLECTIONNEURS-DONATEURS DES MUSÉES DU SÉMINAIRE DE QUÉBEC ET DU MUSÉE DE LA PROVINCE (L'ANCÊTRE DU MUSÉE DU QUÉBEC ET DU MUSÉE DE LA CIVILISATION). [sophie.couturesamson@gmail.com] 
"Maison Henry-Stuart". Répertoire du patrimoine culturel du Québec. Ministère de la Culture, des Communications et de la Condition féminine. $<$ http://www.patrimoineculturel.gouv.qc.ca/RPCQ/> (consulté le 26 août 2008).
Bien qu'elle soit composée majoritairement de citoyens d'origine française, la population du Québec est de plus en plus métissée et multiculturelle. Ainsi, les types de patrimoines sur lesquels les muséologues sont appelés à travailler sont de plus en plus éclatés et touchent diverses communautés culturelles. Parmi celles-ci, la communauté anglophone d'origine britannique (anglaise, écossaise et irlandaise) est héritière d'un legs patrimonial considérable proportionnel à sa population. Et les exemples de sites patrimoniaux et de collections muséales issus de cette communauté sont nombreux. Pensons notamment au Musée McCord d'histoire canadienne, bâti autour de la collection de David Ross McCord, ou à la maison Henry-Stuart, classée Lieu historique national du Canada depuis $1999^{[1]}$.

Ces patrimoines sont bien sûr présents dans les grandes villes que sont Montréal et Québec, mais également dans d'autres régions de la province. Le présent article a pour but de proposer une incursion à Lévis, sur la rive sud de Québec, où nous avons eu l'occasion de faire des recherches concernant l'histoire et le patrimoine de la famille Breakey. Cette famille irlandaise, à l'origine de la municipalité de Breakeyville (aujourd'hui intégrée à la ville de Lévis), s'est installée sur la rive sud de Québec au XIX ${ }^{\mathrm{e}}$ siècle et a œuvré dans le commerce du bois pendant plus de cent ans. Bien que l'essentiel des recherches que nous avons réalisées en lien avec cette famille et son patrimoine consistait en la production d'un inventaire d'une partie de leurs biens immobiliers et mobiliers, et ultérieurement sur une formulation d'options relatives à la conservation et à la mise en valeur de ce patrimoine, nous avons choisi de commencer notre recherche en nous penchant sur quelques considérations théoriques associées à notre sujet. Nous avons conséquemment mené une réflexion sur les liens qui existent entre trois concepts centraux qui se rapportent à notre recherche, soit l'histoire, la mémoire et le patrimoine. 
Nous nous sommes également intéressée au contexte historique dans lequel les Breakey ont évolué. Ce contexte, replaçant les Breakey et le développement de Breakeyville dans la trame historique du commerce du bois sur l'ensemble de la rive sud de Québec, met également en lumière l'histoire d'une famille anglo-protestante qui a fait fortune en employant majoritairement des ouvriers franco-catholiques.

La présentation de ce contexte historique est suivie du résumé des inventaires et des enquêtes réalisés, ainsi que des options de conservation et de mise en valeur retenues pour ce patrimoine. Dans le présent article, nous présentons donc les points saillants de l'étude ${ }^{(2)}$ que nous avons menée pour ainsi faire découvrir une portion de l'histoire et du patrimoine lévisiens.

\section{Baliser l'espace conceptuel :}

\section{histoire, mémoire, patrimoine}

Trouver les raisons pour lesquelles un site, un bâtiment, une collection, peuvent être d'intérêt patrimonial oblige à se questionner sur son histoire, mais également sur son pouvoir d'évocation. Histoire, mémoire et patrimoine s'entrecroisent et s'interpellent. La conception du patrimoine relève de la mémoire, de l'histoire et de l'actuel. C'est-à-dire que le patrimoine, si l'on se réfere à la définition qu'en donne le Groupe-conseil sur la politique du patrimoine culturel du Québec, est le reflet de ce que les gens vivant à une certaine époque choisissent de conserver et de mettre en valeur, parmi les objets hérités des époques précédentes, en raison de l'importance symbolique de ces objets pour une communauté donnée ${ }^{[3]}$. Ces objets historiques, devenus objets patrimoniaux en raison de leur prise en charge par une communauté qui en assure la conservation et la transmission, représentent l'héritage de la communauté. Ils sont le reflet de deux époques : celle d'où provient l'objet patrimonialisé et celle où cet objet historique reçoit son titre d'objet patrimonial. L'histoire est donc essentielle au statut d'objet patrimonial ${ }^{[4]}$. Mais, pour qu'un objet historique devienne un objet patrimonial, il doit être chargé de symbolisme. Autrement dit, il doit être hautement significatif pour la mémoire des communautés contemporaines et contribuer au développement culturel contemporain ${ }^{[5]}$. La mémoire collective, reliée au souvenir (à des lieux, à des objets, à des événements), vient renforcer la mémoire historique, reliée à la production savante. Elle permet d'associer l'histoire au présent et est essentielle à la consécration patrimoniale, au même titre que le patrimoine est essentiel à la mémoire, puisqu'il lui sert de support tangible. L'une ne va pas sans l'autre ${ }^{[6]}$.

\section{[2]}

COUTURE SAMSON, Sophie. Le patrimoine de la famille et des entreprises Breakey. Identification, conservation et mise en valeur. Montréal: Université du Québec à Montréal, 2007. 2 vol.

[3]

Le Groupe-conseil sur la politique du patrimoine culturel du Québec donne cette définition du patrimoine: «Peut être considéré comme 'patrimoine' tout objet ou ensemble matériel ou immatériel, que s'approprie une collectivité en reconnaissant sa valeur de témoignage et de mémoire historique et en faisant ressortir la nécessité de le protéger, de le conserver et de le mettre en valeur." (ARPIN, Roland (dir.). Notre patrimoine, un présent du passé. Québec: Groupe-conseil sur la politique du patrimoine culturel du Québec, 2000, p. 25.)

\section{[4]}

DAVALLON, Jean.

«Tradition, mémoire, patrimoine. In. SCHIELE, Bernard (dir.). - Patrimoine et identités. Québec: Musée de la civilisation, MultiMondes, 2002, p. 45.

[5]

MONTPETIT, Raymond.

«Les musées, générateurs d'un patrimoine pour aujourd'hui. Quelques réflexions sur les musées dans nos sociétés postmodernes". In. SCHIELE Patrimoine et identités, op. cit, p. 79-81.

[6]

Idées de Pierre Nora rapportées dans DROUIN, Martin. Le combat du patrimoine à Montréal (1973-2003). Sainte-Foy: Presses de l'Université du Québec, 2005, p. 9. 


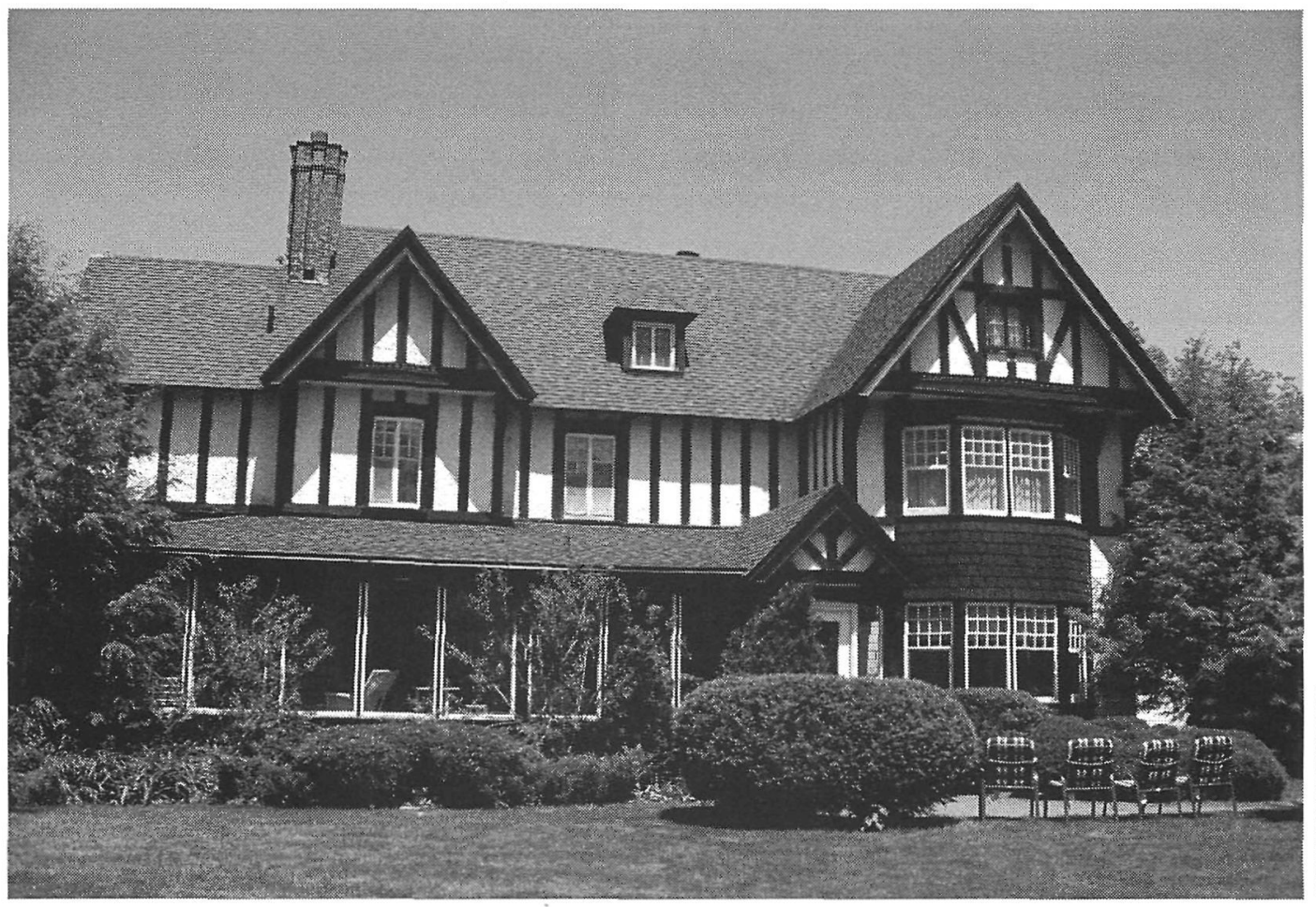

Manoir Breakey

Puisque les vestiges mobiliers et immobiliers des Breakey se trouvent sur le territoire de la ville de Lévis et que la politique culturelle de celle-ci ne comprend aucune définition du patrimoine, nous avons choisi, dans le cadre de notre recherche, de retenir celle qui a été donnée par le Groupe-conseil sur la politique du patrimoine culturel du Québec. Ainsi, le patrimoine de la famille Breakey et de leur entreprise désigne «tout objet ou ensemble matériel ou immatériel, que s'approprie une collectivité en reconnaissant sa valeur de témoignage et de mémoire historique et en faisant ressortir la nécessité de le protéger, de le conserver et de le mettre en valeur» " ${ }^{[7]}$. Pour être qualifié d’objet patrimonial, tout objet historique relié au passé de la famille et des entreprises Breakey doit donc avoir une valeur mémorielle pour les Breakeyvilloises et les Breakeyvillois. Toutefois, puisque la municipalité de Breakeyville, autrefois autonome, fait maintenant partie de la ville de Lévis, la valeur mémorielle nécessaire à la " patrimonialisation " des objets historiques liés aux Breakey doit également toucher les habitants de la ville de Lévis. Connaître l'histoire des Breakey et de leur entreprise et ancrer cette histoire dans celle de la rive sud de Québec est donc une tâche primordiale dans l'identification d'un patrimoine s'y rattachant, tout comme ce patrimoine est un rappel concret de l'histoire et de la mémoire. 
Cadre contextuel : Le commerce du bois

\section{sur la rive sud et le développement de Breakeyville}

Si l'on veut savoir pourquoi le patrimoine de la famille Breakey et de leur entreprise possède un réel intérêt et mérite d'être conservé et mis en valeur, il importe tout d'abord de présenter le contexte historique général dans lequel l'entreprise et la famille ont évolué, ainsi que les thèmes plus spécifiques se rattachant à leur passé. L'histoire, qui permet de donner son poids " académique " au patrimoine, est plus simple à présenter que la mémoire rattachée à cette histoire et à ce patrimoine, puisque aucune enquête n'a été menée auprès de la population de la région pour connaître l'influence des Breakey sur la mémoire collective des habitants. Néanmoins, nous tenterons de présenter les conséquences potentielles de l'histoire sur la mémoire des Lévisiennes et des Lévisiens actuels, par l'importance historique du commerce du bois sur l'ensemble de la rive sud de Québec ${ }^{[8]}$.

Dès le milieu du XVII ${ }^{e}$ siècle, les habitants de la région de Lévis s'adonnent au commerce du bois. Concentré à l'intérieur de la colonie, ce commerce sert principalement au chauffage et à la construction, notamment à la construction navale. Bien que les exportations de bois soient quasi absentes aux XVII et XVIII e siècles, l'importance de cette activité économique sur le territoire lévisien, à cette époque, ne doit pas être sous-estimée ${ }^{\left[{ }^{[0}\right.}$. Notons entre autres que, sous le Régime français, les ursulines font construire un moulin à la rivière à la Scie. Au XVIII ${ }^{e}$ siècle, Gilles William Strouds et Jacques Lafontaine de Belcour, d'importants entrepreneurs de l'époque, font eux aussi construire des moulins sur la rive sud. Ils sont d'ailleurs les premiers à tirer profit de l'anse de New Liverpool pour diverses activités autour du commerce du bois (bois de chauffage, bois de charpente, activités navales) ${ }^{[10]}$.

Au début du XIX ${ }^{e}$ siècle, un intérêt extérieur commence à se faire sentir pour le bois canadien, principalement en raison du blocus napoléonien de 1804 et de l'épuisement des forêts anglaises. La Grande-Bretagne se tourne donc vers sa colonie, ce qui entraîne le développement de l'exportation du bois ainsi que l'expansion du port de Québec (à la fois sur la rive nord et sur la rive sud), permettant à Lévis de tirer profit des retombées économiques de ce nouveau commerce.

À cette époque un groupement de bourgeois, les Lumber Lords de Québec (dont font partie William Price, James Patton, James Tibbits et Allan Gilmour), s'organise et prend avantage du littoral lévisien et ouvre des chantiers dans les anses les plus propices au flottage et à l'entreposage du bois. Si cette industrie permet une expansion portuaire, elle impulse également une expansion urbaine dans la région de Québec, l'augmentation du nombre de travailleurs nécessaires dans les ports et les anses à bois entraînant l'accroissement
[8]

Id., p. 23.

[9]

SAMSON, Roch (dir.).

Histoire de Lévis-Lotbinière.

Québec: Institut québécois de recherche sur la culture, 1996, p. 145; COURVILLE, Serge. Le Québec. Genèse et mutations du territoire. Synthèse de géographie historique. Sainte-Foy: Presses de I'Université Laval, 2000, p. 217. [10]

PRITCHARD, James S.

«Strouds, Gilles William *. Dictionnaire biographique du Canada en ligne. Dictionnaire biographique du Canada. <http://www.biographi.ca/> (consulté le 4 mai 2007); LEMIEUX, Jacques. La maison Malakoff. Saint-Romuald, 1998, p. 39-45. 
[12]

SAMSON,

op.cit, p. 155

et 189 ; COURVILLE, op.cit, p. 218.

[13]

SAMSON,

op.cit, p. 348; COUTURIER,

Jacques-Paul. Un passé composé. Le Canada de 1850 à nos jours. Moncton: Éditions d'Acadie, 2000, p. $82-86$.

[14]

SAMSON,

op.cit, p. 339; BERTHOLD,

Étienne. Lévis. Sainte-Foy:

Éditions de l'IQRC (Institut québécois de recherche sur la culture), 2006, p. 101-103.

[15]

SAMSON, op.cit, p. 585.

[16]

RENY BOURGET, Paule. Sainte-Hélène-de-Breakeyville: d'hier à aujourd'hui. Sainte-Hélène-de-Breakeyville: Comité des fêtes de Sainte-Hélène-de-Breakeyville, 1984 , p. 470 et 475.

[ 7 ]

Id., p. $479,482,502,506$ et 510 . du nombre d'habitants à Québec et à Lévis. Rappelons ici que les employeurs sont alors majoritairement issus de la minorité anglo-protestante qui habite Québec et sa rive sud et que les travailleurs sont principalement des Canadiens-français ${ }^{[11]}$.

Importante source d'emplois et de revenus, l'industrie du bois permet également l'accroissement de la construction navale à Québec et à Lévis, qui entraîne à son tour l'implantation de nouvelles industries, dont des fonderies. Construction navale et industrie du bois sont interconnectées et contribuent grandement au dynamisme économique de la région.

En 1850, le secteur des anses lévisiennes foisonne de chantiers et est un important lieu d'activités économiques pour l'ensemble de la région de Québec. L'installation du terminus du Grand Tronc à l'anse Tibbits, à Lévis, en 1854 , ajoute à cette effervescence. À cette époque, Lévis est donc en voie de dépasser Québec en tant que deuxième ville économique en importance au Québec, après Montréal ${ }^{[12]}$.

La crise économique de 1873 frappe cependant durement la rive sud. Elle y entraîne morosité et émigration et ce, jusqu'à la fin du siècle. Le commerce du bois et la construction navale vont presque disparaître. Seules les industries les plus fortes vont survivre, dont les moulins Atkinson et John Breakey Limited ${ }^{[13]}$. La fin du XIX ${ }^{\mathrm{e}}$ siècle voit également arriver la machine à vapeur, puis l'électricité, qui vont tranquillement remplacer l'eau comme source d'énergie et déplacer plusieurs industries de la campagne vers la ville ${ }^{[14]}$.

Après 1900, les pâtes et papiers vont presque complètement remplacer le bois de sciage dans la région. Les entreprises réorientent donc leurs activités. Elles sont cependant fortement touchées par la crise économique de 1929. Seule la John Breakey Limited va perdurer au-delà de la crise des années 1930 et traverser la quasi-totalité du XX ${ }^{\mathrm{e}}$ siècle ${ }^{[15]}$.

C'est d'abord dans une anse à bois qu'aurait travaillé Hans Denaston Breakey, à son arrivée d'Irlande, dans les années 1830 . En 1846, il se lance, avec son associé Charles King, dans la construction d'une scierie le long de la rivière Chaudière ${ }^{[16]}$. Après le décès de Charles King et de Hans Denaston Breakey, leurs descendants vont les remplacer à la direction de l'entreprise. Progressivement, les descendants de Charles King se retirent cependant. L'entreprise se retrouve donc uniquement entre les mains des Breakey, à partir de 1878. Sous la présidence de John Breakey, la compagnie acquiert son propre chemin de fer, la Chaudière Valley Railway, et construit un deuxième moulin, alimenté par la vapeur plutôt que par l'eau ${ }^{[17]}$.

Bien que quelques habitants soient déjà présents sur le territoire de la future municipalité de Breakeyville au début du XIX ${ }^{\mathrm{e}}$ siècle, c'est l'implantation du moulin de la King and Breakey en 1847 qui contribue 
le plus à faire augmenter la population de ce secteur et qui va entraîner la fondation de la paroisse et de la municipalité de Breakeyville. C'est d'ailleurs John Breakey, fils de Hans Denaston, qui défraie les coûts de construction de l'église de Sainte-Hélène-de-Breakeyville en 1909. Il est intéressant de constater. qu'une famille protestante ait pu payer pour la construction d'une église catholique destinée à ses ouvriers et à leurs familles, alors qu'eux-mêmes, à cette époque, n'avaient pas encore de lieu de culte à proximité (l'église protestante de Breakeyville sera construite en 1927). Impliquée dans la création de la paroisse et de la municipalité, la famille Breakey va également participer à la vie municipale. Deux de ses membres, Denaston Breakey et John Archibald Scott, vont être maire de la ville durant quelques années ${ }^{[18]}$.

Euvrant principalement dans le commerce du bois scié, la compagnie John Breakey Limited réoriente ses activités en 1921, sous la présidence de Colin Cathcart Breakey, pour se tourner vers l'écorçage du bois de pulpe exporté vers les papetières. Touchée par la crise économique des années 1930, la compagnie ferme temporairement ses portes de 1930 à 1932. Dans les années 1940, elle emploie une centaine d'hommes à Breakeyville et jusqu'à 2000 autres dans les chantiers de coupe. Elle réoriente à nouveau ses activités en 1947 pour produire dorénavant de la pâte à papier et construit un nouveau moulin à pâte mécanique qui est exploité à partir de 1949. La présidence de l'entreprise passe ensuite de Colin Cathcart Breakey à son beau-frère, John Archibald Scott, puis aux fils de ce dernier, Jim et Rex Scott ${ }^{[19]}$.

Enfin, le 10 octobre 1981, soit 134 ans après la construction du premier moulin par Hans Denaston Breakey et Charles King, la John Breakey Limited cesse ses activités en raison de la chute du marché de la pâte entraînée par la récession économique. En 1984, l'usine est achetée par Papier Cascades Inc. et fonctionne encore aujourd'hui sous le nom de Fibres Breakey inc.

La famille et les entreprises Breakey s'insèrent donc dans le contexte historique du développement économique de Lévis au cours du XIX ${ }^{e}$ siècle. Le commerce du bois, auquel les entreprises Breakey sont apparentées, est une activité primordiale de l'économie lévisienne durant ce siècle.

Puisque la famille et les entreprises Breakey sont au cœur du développement et de la vie économique de la paroisse et de la municipalité de Sainte-Hélène-de-Breakeyville, et bien qu'aucune enquête n'ait été menée auprès des habitants de cette ancienne municipalité, il est évident que les Breakey et leur histoire font partie intégrante de la mémoire de la population de ce secteur. De plus, en raison de la renommée, de l'importance des entreprises Breakey et de leur lien direct avec le commerce du bois sur la rive sud de Québec, leur histoire fait également partie de la mémoire des habitants de Lévis. Histoire et mémoire se conjuguent donc pour faire des Breakey une portion incontournable de l'héritage lévisien. 
Ressources patrimoniales liées à la famille Breakey

et à leur entreprise

Dans le cadre de notre travail de recherche, seulement quelques éléments du patrimoine de la famille Breakey ont été repérés et inventoriés. Cependant, nous avons été en mesure d'identifier d'autres éléments patrimoniaux, qui seront présentés plus loin dans le présent article et qui mériteraient eux aussi de faire l'objet d'un inventaire et d'une étude. Les éléments que nous avons inventoriés et étudiés ici sont les lieux de mémoire de la famille Breakey, la collection de livres de Louise Breakey et Colin Cathcart Breakey, ainsi que le récit de vie de Dorothy Higgins. Nous avons conséquemment procédé à l'identification, à l'inventorisation et à l'étude de ces éléments patrimoniaux.

En ce qui a trait au patrimoine matériel de la famille Breakey, notre recherche s'est tout d'abord tournée vers les lieux de mémoire de la famille et des entreprises Breakey. Nous avons classé ces lieux que nous avons inventoriés - au nombre de $\mathbf{1 4 9}$ - sous six groupes distincts : les lieux religieux, le domaine Breakey, la vie communautaire, le chantier et les deux quartiers ouvriers.

Les dates de construction et les secteurs où se trouvent les maisons nous ont permis d'identifier deux période de forte construction domiciliaire, soit 1909-1910, époque de fondation de la paroisse (premier quartier ouvrier), et 1947-1948, date de construction du moulin à pâte mécanique (second quartier ouvrier). Le domaine Breakey est constitué des maisons qui ont appartenu aux membres de la famille Breakey et sont des symboles de la réussite économique de la famille, mais également de la domination de cette famille anglo-protestante sur la population franco-catholique de la paroisse. Les lieux religieux sont quant à eux composés principalement du cimetière, de l'église catholique, des vestiges de l'église protestante ainsi que du presbytère de Breakeyville, évoquant l'histoire spirituelle de la communauté marquée par une population francophone et anglophone; catholique et protestante. La vie communautaire est évoquée dans notre inventaire par quelques bâtiments ayant servi d'épicerie, de magasin, de banque, de bureau de poste, etc. Ces édifices ont, pour certains, conservé leur vocation commerciale et sont tous situés le long de la rue principale de Breakeyville, près des quartiers ouvriers, du domaine Breakey et des moulins. Enfin, l'ensemble des bâtiments liés au chantier est composé de la friche du premier moulin ainsi que du moulin mécanique et s'amalgame aux quartiers ouvriers pour illustrer l'activité industrielle de la ville.

Par leur appartenance à l'un de ces ensembles, tous les lieux étudiés sont symboliques pour l'histoire des Breakey, mais également pour celle de 


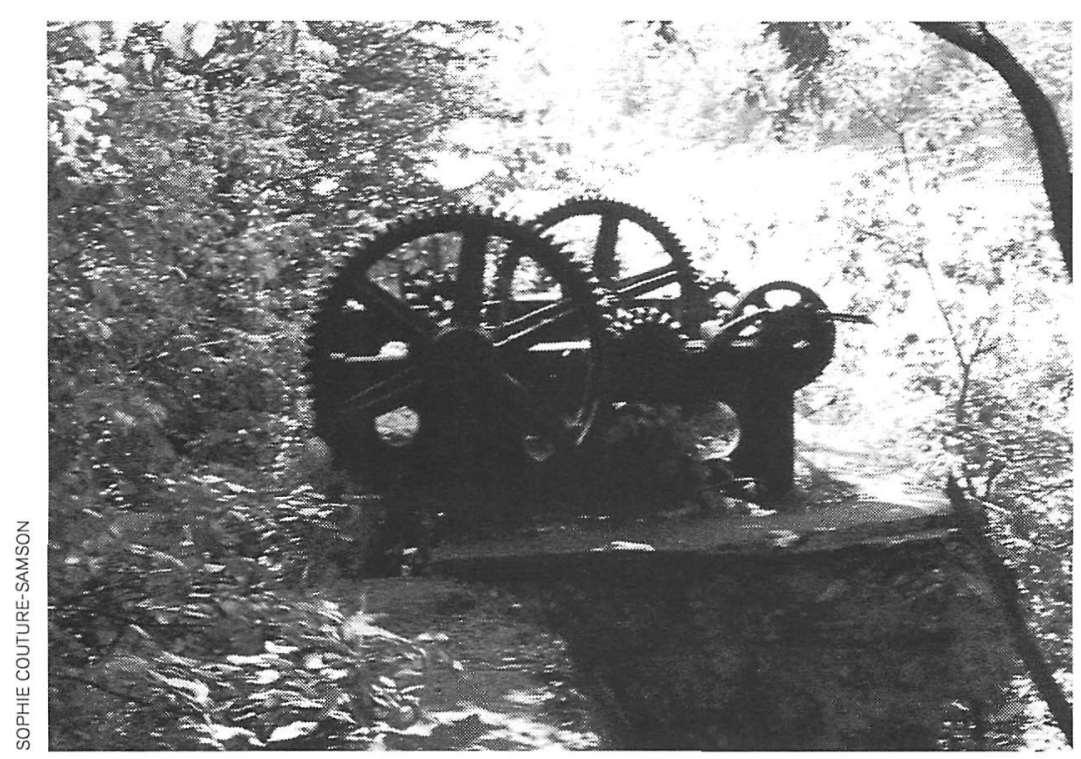

Friche du premier moulin

la municipalité de Breakeyville et ils sont d'importants supports mémoriels pour la communauté. Chacun de ces lieux de mémoire est, de toute évidence, associé à l'histoire de la famille et des entreprises Breakey, puisque c'est en raison de l'implantation des scieries Breakey dans ce secteur que la paroisse et la municipalité ont pu se développer. Mais ces lieux de mémoire sont également très fortement liés à la vie municipale et communautaire de Breakeyville et au passé de chacune des familles qui ont habité ces maisons, ont fréquenté l'église ou les magasins de Breakeyville et ont travaillé dans l'un ou l'autre des moulins. L'histoire des Breakey, de Breakeyville et de ses habitants, passés et présents, est intimement interreliée et les supports mémoriels que sont les bâtiments inventoriés lors de notre étude en sont des exemples tangibles.

Toujours sur le plan du patrimoine matériel, outre les objets patrimoniaux immobiliers, nous avons eu la chance d'inventorier des objets patrimoniaux mobiliers, soient les livres gardés à la bibliothèque de Colin Cathcart Breakey et Louise Breakey, aujourd'hui possédés par Agathe et Roger Lachance. En tout, ce sont 715 livres qui ont été répertoriés et catalogués. Il s'agit majoritairement de romans, souvent d'œuvres " populaires », ayant été des best-sellers à l'époque de leur parution ou encore ayant été adaptés pour en faire des films, des pièces de théâtre ou des comédies musicales sur Broadway. Nous y trouvons des livres en anglais uniquement, écrits par plus de 300 auteurs différents. Parmi eux quelques-uns se démarquent, du fait que plusieurs de leurs livres y sont présents (Philip Gibbs, Vicki Baum, Robert Hichens, William J. Locke, Rafael Sabatini et Mazo de la Roche). L'année 
[20]

MICHON, Jacques (dir.).

Histoire de l'édition littéraire au Québec au XXe siècle. La naissance de l'éditeur, 1900-1939, volume 1 .

Saint-Laurent: Fides, 1999, p. $96-98$ et $103-104$.

[21]

ROBERGE, Martine. Guide d'enquête orale. Québec: Publications du Québec, 1991, p. 12,14 et 19 . d'édition de ces livres se situe de 1888 à 1955, mais la majorité de ceux-ci sont parus durant le deuxième quart du $\mathrm{XX}^{\mathrm{e}}$ siècle. Nous avons également noté une forte proportion de livres parus en 1930 et 1931, époque à laquelle Colin Cathcart Breakey a dû fermer temporairement la compagnie, ce qui lui a probablement laissé davantage de temps pour s'adonner à la lecture.

Si l'on se rapporte au contexte historique et littéraire québécois de la première moitié du $\mathrm{XX}^{\mathrm{e}}$ siècle, on remarque que la collection de Louise et Colin Cathcart Breakey est assez représentative de l'époque. Le fait qu'on y trouve des livres dits "populaires " est assez caractéristique de la production littéraire de divertissement qui vient s'ajouter à la littérature «lettrée» sur les rayons des librairies au tournant du $\mathrm{XX}^{e}$ siècle. Les librairies ne s'adressent désormais plus uniquement à une élite intellectuelle (universitaires, clergé, médecins, etc.): elles visent désormais aussi le grand public. Il n'est pas non plus surprenant de constater que la majorité des livres des Breakey proviennent des États-Unis et de la Grande-Bretagne, puisque ces pays sont à l'origine de presque toutes les importations de livres au Canada, avec $91 \%$ des importations totales en 1910. Les deux guerres mondiales vont par ailleurs contribuer à augmenter le débit des importations américaines, en raison du ralentissement de celles en provenance de l'Europe. Avec plus de 50\% de livres édités et imprimés aux États-Unis et plus de $20 \%$ en Grande-Bretagne, la collection de Louise et Colin Carthcart Breakey est assez caractéristique du contexte duquel elle est issue ${ }^{[20]}$.

Comme peu d'objets ayant appartenu à la famille Breakey existent encore de nos jours, les livres de Colin Cathcart et Louise Breakey représentent une part importante du patrimoine mobilier de cette famille et sont un exemple assez représentatif de la littérature anglophone du début du $\mathrm{XX}^{\mathrm{e}}$ siècle.

$\mathrm{Au}$ volet du patrimoine immatériel, notre travail nous a permis de réaliser une entrevue avec Dorothy Higgins, qui a été à l'emploi des Breakey durant de nombreuses années. Son témoignage est unique, puisqu'elle est la seule personne encore vivante à avoir exercé le poste d'aide domestique et de surintendante auprès de la famille Breakey. Ce qui est le plus intéressant dans le témoignage qu'elle nous a livré, c'est la présentation qu'elle nous a fait de l'histoire telle qu'elle l'a vécue et perçue. La valeur de son témoignage ne réside pas dans son potentiel informatif, mais plutôt dans sa perception des faits et du discours sur le passé, discours teinté de ses expériences, de ses valeurs, de sa vision de ce qui est arrivé. Dorothy Higgins nous a livré sa version du passé, sa mémoire; une mémoire unique qui enrichit le patrimoine des Breakey et qui doit être conservée, tout comme celle des autres travailleurs qui ont œuvré pour les Breakey ${ }^{[211}$. De moins en moins nombreux, ces travailleurs constituent la mémoire vivante d'une ville et d'une entreprise plus que centenaire qui a marqué le visage de la rive sud de Québec. Le fait de conserver et d'étudier les récits de ces travailleurs pourrait nous permettre 
de mettre en lumière les relations entretenues entre les employeurs et les employés, cette minorité d'anglophones qui dominait la population francophone de la ville. Il s'agit également d'un moyen de mieux connaître la population ouvrière de Breakeyville.

Certes, les éléments patrimoniaux que nous avons étudiés lors de cette recherche ne forment pas l'ensemble du patrimoine de la famille et des entreprises Breakey existant toujours en 2008. Il est donc essentiel, dans les prochains mois et les prochaines années, de poursuivre les recherches concernant l'histoire et le patrimoine des Breakey, par exemple en réalisant d'autres enquêtes auprès de nouveaux informateurs, en étudiant les livres comptables des industries Breakey conservés à la Société historique de Breakeyville, en interrogeant la population de Breakeyville quant aux photos, documents, objets, etc. qu'ils pourraient posséder et qui pourraient faire partie du patrimoine des Breakey et de leur entreprise. Ce ne sont ici que quelques-unes des pistes de développement qui s'offrent à nous quant à la poursuite de l'identification de ce patrimoine.

\section{Conservation du patrimoine}

Au volet de la conservation des éléments patrimoniaux étudiés, nous avons retenu diverses possibilités. Tout d'abord, pour ce qui est du patrimoine immobilier, c'est-à-dire les lieux de mémoire que nous avons identifiés, nous recommandons la constitution d'un site du patrimoine. Puisque le patrimoine de la famille et des entreprises Breakey est d'importance municipale et régionale, les statuts nationaux que sont le classement, la reconnaissance ou le décret d'arrondissement historique ne peuvent s'appliquer au cas qui nous intéresse ici. Comme les lieux de mémoire répertoriés sont nombreux, la constitution d'un site du patrimoine est plus judicieuse que la citation de monuments historiques ${ }^{[22]}$.

Ce site du patrimoine ne comprendrait pas l'ensemble de l'ancienne municipalité de Breakeyville, mais un secteur précis de son territoire comportant la rue principale de Breakeyville (avenue Saint-Augustin) ainsi que les cinq ensembles présentés précédemment, soit le domaine Breakey, les lieux religieux, le chantier et les deux quartiers ouvriers.

Au sujet du cas précis qu'est la friche du premier moulin de la King and Breakey, qui fait partie de l'ensemble «chantier " de notre inventaire des lieux de mémoire, nous recommandons le nettoyage du site et l'installation de panneaux d'interprétation présentant le lieu comme un site patrimonial. Nous pensons également que l'endroit possède un bon potentiel archéologique ${ }^{[231}$,
[22]

La Loi sur les biens culturels. Guide pratique destiné aux municipalités. Québec: Ministère de la Culture et des Communications, 2006, p. 9-11 et 18-19.

[23]

Le site présente en effet de nombreux murs de béton ainsi que quelques pièces d'équipement. Le nettoyage du secteur pourrait permettre de mettre au jour les vestiges du premier moulin et peut-être même de trouver d'autres pièces d'équipement enfouies. 
qu'il serait pertinent de faire évaluer le véritable potentiel du secteur et, $s^{\prime}$ il y a lieu, d'y effectuer des fouilles archéologiques.

Enfin, pour conclure nos recommandations relatives au patrimoine immobilier, nous suggérons que la Ville de Lévis produise un petit guide à l'usage des citoyennes et des citoyens, où seraient présentés divers trucs et conseils concernant la conservation et la restauration de bâtiments historiques. Ce guide serait profitable non seulement aux habitants de Breakeyville (dont la demeure est répertoriée dans notre inventaire des lieux de mémoire), mais également à tous les propriétaires d'une maison ancienne qui habitent la ville de Lévis.

Pour ce qui est des livres de Louise et Colin Cathcart Breakey, qui se trouvent encore dans leur lieu d'origine (la bibliothèque du manoir Breakey), nous avons recommandé, pour assurer leur conservation à long terme, qu'ils soient transférés dans un endroit plus approprié. Bien sûr, la collection n'est pas en danger immédiat, puisque ses propriétaires sont conscients de sa valeur historique et patrimoniale. Toutefois, bien qu'Agathe et Roger Lachance prennent soin de la collection, la pièce où elle se trouve n'est pas adéquate, elle est même néfaste à la conservation des livres; entre autres nous y avons constaté d'importantes variations de chaleur et d'humidité et la lumière du jour qui entre par les fenêtres contribue à la dégradation de la pigmentation des couvertures et des reliures. Nous avons fait la recommandation de transférer les livres dans un local appartenant à la Société historique de Breakeyville, qui est à l'épreuve du feu et de l'humidité et dont l'accès est contrôlé. Cependant, à long terme, l'idéal serait de placer cette collection dans une véritable réserve muséale, qui servirait à conserver uniquement les objets patrimoniaux de la ville de Lévis. Nous avons donc recommandé également la mise sur pied d'une telle réserve afin d'assurer une meilleure conservation du patrimoine des Breakey, mais également de l'ensemble du patrimoine matériel lévisien.

Nos recommandations concernant le patrimoine immatériel des Breakey ont quant à elles porté sur la réalisation de nouvelles entrevues avec des porteurs de patrimoine - comme celle faite auprès de Dorothy Higgins - qui pourraient nous renseigner sur leurs liens avec les Breakey, que ce soit en tant que membres de la famille, amis, employeurs, etc. Comme plusieurs de ces témoins de l'histoire des Breakey ont atteint un certain âge, il est primordial de dresser rapidement une liste des informateurs potentiels et de commencer le plus tôt possible les entrevues avec les personnes concernées pour recueillir le plus de témoignages possible. D’autres entrevues pourraient ensuite avoir lieu avec des informateurs plus jeunes ou avec des groupes d'informateurs qui pourraient discuter ensemble de leurs expériences personnelles. 


\section{Mise en valeur du patrimoine}

Avant la réalisation de notre étude, l'interprétation et la mise en valeur à Breakeyville étaient limitées à une piste cyclable construite sur le tracé qu'empruntait autrefois la Chaudière Valley Railway dans le secteur de Breakeyville. Cette piste comprenait un parcours interprétatif, intitulé "Au temps des Breakey", abordant divers thèmes qui touchent à leur histoire: moulins, chemin de fer, grands événements de l'histoire de la municipalité, etc. Toutefois, la piste cyclable nous a semblé peu fréquentée. Ainsi, bien que le parcours interprétatif soit de bonne qualité, sa visibilité est-elle restreinte. Il importe donc, selon nous, de trouver des solutions pour diffuser le patrimoine des Breakey auprès d'une portion beaucoup plus grande de la population.

Dans le but de trouver des idées de mise en valeur s'adaptant bien à un site de patrimoine industriel, nous avons consulté divers documents et sites Internet se rapportant à trois principaux sites, soit la ville de Jonquière, la cité ouvrière de Noisiel ainsi que le Lieu historique national des Forges-duSaint-Maurice. Cette consultation nous a permis de nous familiariser avec leurs activités d'interprétation et d'élaborer des options de mise en valeur pour Breakeyville.

Nous avons conséquemment formulé plusieurs recommandations à court, moyen et long terme concernant la mise en valeur du patrimoine des Breakey. À court terme, notre choix s'est arrêté sur la diffusion des divers lieux de mémoire inventoriés par le biais du journal municipal de Lévis. De courts articles pourraient être réalisés à partir de quelques-uns des lieux de mémoire les plus significatifs, ou les mieux conservés, en présentant leur histoire ainsi que leur intérêt patrimonial. Issue de la documentation concernant la ville de Jonquière, cette idée d'articles présentant divers lieux de mémoire a entre autres pour avantages d'être rapide à réaliser et d'engager peu de frais ${ }^{[24]}$.

À moyen terme, les activités de mise en valeur pourraient entre autres prendre la forme d'une exposition itinérante de la collection de Louise et Colin Cathcart Breakey. Cette exposition, qui présenterait quelques-uns des livres et des auteurs présents dans la collection, pourrait circuler dans les bibliothèques publiques de Lévis.

Une autre façon, à moyen terme, de mettre en valeur le patrimoine des entreprises Breakey serait de créer un site Internet qui permettrait aux internautes de faire une visite virtuelle de Breakeyville, en plus de leur fournir de l'information sur l'histoire de Breakeyville et de remettre l'implantation et l'histoire des entreprises Breakey dans le contexte historique lévisien. Enfin, le nettoyage et l'aménagement de la friche du premier moulin de la

\section{[24]}

NOPPEN, LUC et LUcie K. MORISSET.

Rapport sur la conservation du patrimoine bâti de Jonquière. Sainte Foy: La Renaissance, 1994, p. 21. 
King and Breakey pourraient également être réalisés à moyen terme et être accompagnés de l'installation d'une plaque commémorative rappelant le $160^{\mathrm{e}}$ anniversaire de construction du moulin et de l'implantation des entreprises Breakey.

Finalement, l'établissement d'activités de mise en valeur à long terme dépend largement des fonds disponibles et de l'avancement des travaux de recherche, de conservation et de mise en valeur du patrimoine lévisien. Nous croyons qu'il serait souhaitable de procéder à la création d'un musée consacré à la présentation d'expositions sur divers sujets liés à l'histoire de la ville de Lévis et de sa région immédiate. La construction d'un tel musée s'accorde avec notre recommandation concernant la création d'une réserve muséale pour entreposer le patrimoine de la ville. Cependant, la mise sur pied d'une réserve est selon nous plus pressante que la création du musée, puisque, outre le patrimoine des Breakey, d'autres patrimoines lévisiens pourraient disparaître si la Ville ne réagit pas rapidement. Une fois le patrimoine protégé dans une réserve, un projet de musée serait réalisable et souhaitable puisqu'il permettrait de faire découvrir l'histoire de Lévis, non seulement aux Lévisiennes et aux Lévisiens, mais également à l'ensemble de la population québécoise. Ce musée, englobant l'ensemble de l'histoire de Lévis, pourrait ainsi toucher divers thèmes (histoires sociale, militaire, culturelle, religieuse, économique, industrielle, etc.) et tisser des liens entre chacune des anciennes municipalités formant la nouvelle ville de Lévis.

Comment les Breakeyvilloises, les Breakeyvillois, les Lévisiennes et les Lévisiens perçoivent-ils l'histoire des Breakey et la mise en valeur du patrimoine issu de cette famille et de leur entreprise? Nous ne pouvons nous prononcer officiellement sur cette question pour l'instant puisque, comme nous l'avons déjà mentionné, aucune enquête n'a été menée pour tâter le pouls de la population. Cependant, par les contacts que nous avons eus avec plusieurs habitants actuels de Breakeyville lors de nos recherches et de notre inventaire des lieux de mémoire de la famille et des entreprises Breakey, nous sommes portée à croire que ce patrimoine est synonyme de fierté pour la communauté de laquelle il est issu.

Pour les Lévisiennes et les Lévisiens, le patrimoine des Breakey, qui peut être jumelé au patrimoine des Davie (Lieu historique national du Canada du Chantier A.C. Davie), rappelle l'époque à laquelle Lévis et son littoral étaient d'importants lieux d'activité économique où foisonnaient les chantiers navals, les anses à bois, les trains partant vers les États-Unis ou vers Montréal, etc. Ce patrimoine permet de faire revivre un âge d'or aujourd'hui oublié par une grande partie de la population.

Pour les Breakeyvilloises et les Breakeyvillois, touchés directement par la famille et les entreprises Breakey, ce patrimoine est également celui 
de la plupart des familles vivant dans ce secteur et qui ont tous un père, un grand-père ou un ancêtre qui a travaillé pour les scieries Breakey. C'est également le patrimoine municipal de la population qui doit son enracinement sur les bords de la rivière Chaudière à l'implantation et à la croissance, pendant plus de cent ans, des entreprises Breakey. Bien sûr, mettre en valeur le patrimoine de cette famille c'est mettre à l'avant-plan le patrimoine d'une minorité bourgeoise anglophone, mais ce patrimoine n'est pas seulement celui de la minorité. Dans le cadre de la recherche que nous avons menée, seuls les livres ayant appartenu à Louise et Colin Cathcart Breakey sont une portion de patrimoine se rattachant uniquement (ou presque) à la famille. Le patrimoine immobilier ainsi que le patrimoine immatériel sont à la fois le reflet de cette famille bourgeoise anglophone et de la population ouvrière francophone de Breakeyville.

Maintenant que les entreprises Breakey n'existent plus, que les descendants de cette famille voisinent les anciens ouvriers et les descendants d'anciens ouvriers des scieries et que les villas bourgeoises ayant appartenu aux Breakey sont entre les mains de familles francophones, on peut dire que la situation a beaucoup changé. La minorité anglophone et la majorité francophone sont unifiées et l'histoire et la mémoire des Breakey et de Breakeyville sont tellement liées que le patrimoine s'y rattachant ne peut être aisément divisé entre l'un et l'autre. La reconnaissance du patrimoine des Breakey et de leur entreprise est peut-être en partie la mise en valeur de l'héritage d'une minorité ethnique dominante par une majorité jadis dominée, mais c'est aussi la reconnaissance de l'histoire del'ensemble d'une municipalité et de sa communauté.

Ainsi, les objets historiques liés aux Breakey et à leur entreprise sont également des objets historiques associés à l'histoire de la communauté et de l'ancienne municipalité de Breakeyville. Ces objets sont donc le reflet d'une époque révolue : celle de la domination des Breakey et du commerce du bois dans la région, celle de l'autonomie de la municipalité de Breakeyville. Mais ils sont également le reflet de la société contemporaine qui choisit de s'y intéresser et de les conserver puisqu'ils sont des supports tangibles d'une mémoire collective. 


\section{Summary}

\section{The Heritage of the Breakey Family and Business:}

\section{Identification, Preservation and Development}

[Translated by Andrea Hutchinson]

[25]

BERGERON Yves and

LUC DUPONT. "Essai sur

les tendances dans les musées de société. Le cas du

Musée de la civilisation*. In.

BERGERON, Yves (dir.),

Musées et muséologie.

Nouvelles frontières. Essais

sur les tendances. Québec:

Musée de la civilisation,

Montréal: Société des

musées québécois,

2005, p. 130-131.

[26]

Ville de Lévis.

Politique

culturelle de Lévis

Lévis, 2004, p. 7.

[27]

Since the municipal mergers, the city of Lévis integrates the former municipalities of Lévis,

Pintendre, Saint-Joseph-de-la-

Pointe-de-Lévy, Saint-Romuald,

Saint-Jean-Chrysostome,

Charny, Sainte-Hélène-

de-Breakeyville, Saint-

Rédempteur, Saint-Nicolas, and Saint-Étienne.
As highlighted by Yves Bergeron and Luc Dupont in the collective work entitled Musées et muséologie. Nouvelles frontières, since the beginning of the 1990s, governments have increasingly tended to develop cultural and heritage protection policies. The Quebec government, for that matter, adopted its own cultural policy in 1992. In the wake of the adoption of that policy and according to governmental recommendations, many Quebec municipalities also established cultural policies. ${ }^{[25]}$ In 2004, the city of Lévis adopted its cultural policy with the main objective of reinforcing its citizens' sense of pride and belonging through the knowledge, protection, development, and transmission of the City of Lévis' heritage-related wealth. ${ }^{[26]}$

Our work, carried out in cooperation with the City of Lévis' Service des arts et de la culture, falls within that intention of reinforcing the citizens' sense of belonging in the new city. ${ }^{[27]}$ Our research focuses on the Breakey family, who was active in the wood business on the south shore of Quebec City for over one hundred years and who was behind the founding of the municipality of Sainte-Hélène-de-Breakeyville, today merged with the Chutes-de-la-Chaudière-Est district of the city of Lévis. In order to comply with the objectives of the City's cultural policy, we mainly address the problem of how and why the heritage related to the Breakey family and their business should be preserved and developed. We thus summarize the theoretical considerations surrounding our topic and the historical context linked to the Breakeys, before presenting the heritage inventory related to the Breakeys, the ethnological survey, as well as the preservation and development options that were selected.

After defining the concepts of history, memory, and heritage, and establishing the interconnections essential to the definition and identification of the Breakey's family and business heritage, we describe the Breakey's history, rooted in Lévis and Quebec City's south shore, in order to understand how these histories known as "scholarly" are interrelated, and how they are integrated in the collective memory and heritage. These historical and theoretical considerations help us to comprehend the reasons why it is important for the City of Lévis be involved in the safeguard and valorization of the heritage of the Breakey family and business. 
We then identify some noteworthy elements related to heritage that we inventoried, i.e., the Colin Cathcart Breakey and Louise Breakey Library, as well as various historical buildings that can be found in the district of the former municipality of Sainte-Hélène-de-Breakeyville. At a more immaterial level, we also carried out an ethnological survey with Dorothy Higgins, who worked for many years as a housekeeper for the Breakeys. The study and identification of these immaterial and material heritage elements allow us to review their current preservation and development stages, and to issue recommendations concerning the safeguard and transmission of this industrial heritage of regional interest.

Buildings, objects, and archive documents have already been lost. Although the citizens of Breakeyville have taken care of and preserved several elements of their material heritage, these may sink into oblivion (as they are disseminated in the municipality's aging population). This heritage nevertheless deserves to be preserved and it and requires short-, medium- and long-term actions to that effect. 